\title{
\#Teeth\&Tweets; The reach and reaction of an online social media oral health promotion campaign
}

Gemma Potts BA (Hons) MRes

Outreach Officer

University of Portsmouth Dental Academy, Faculty of Science, University of Portsmouth.

Portsmouth PO1 2QG

Email gemma.potts@port.ac.uk

David R Radford BDS PhD FDS MRD

Reader in Integrated Dental Education and Multi-professional Care

King's College London Dental Institute at Guy's, King's College \& St Thomas' Hospitals

St Thomas' Street, London Bridge, London SE1 9RT

and the University of Portsmouth Dental Academy, Faculty of Science, University of Portsmouth. Portsmouth PO1 2QG

Email david.radford@kcl.ac.uk

Key words: Oral health promotion, oral health inequalities, Twitter, social media 
Aim The aim of the study was to investigate: (i) the geographical reach and reaction of the online participants engaging in an oral health campaign 'National Smile Month' UK 2016 (NSM) and (ii) whether dental practices during NSM were using Twitter to help address regional oral health inequalities. Methods Twitter posts (i.e. 'tweets') were collected using the API (application programming interface) software Mozdeh, for one month. Tweets were classified into high, medium or low engagement. Participants' postcode data of the organisation/practice were obtained via an internet search using Google. The geolocation of tweets was then linked by organisations' postcode to the 2015 Index of Multiple Deprivation and the oral health survey of 5 year olds 2014/15 and mapped using Google Fusion Tables. Results A total of 23,100 tweets were captured with a final total of 2968 usable tweets from 763 separate accounts. 212 of tweets were from dental practices. 107 were classified as low engagement, 99 medium engagement and 47 high engagement. Interactive maps were created to give a visual representation of the relationship between those participants producing 'high' impact tweets and the level of dental decay in 5 year olds and deprivation levels. Conclusion The majority of tweets did not promote any specific preventative behaviour. Dental practices in England were not contributing to National Smile Month via Twitter in a way that would improve regional oral health inequalities. In areas of high need there is evidence of pro-active engagement with NSM via Twitter by Local Authorities and their health care partners.

\section{In Brief Box}

- Twitter has the potential to be a useful tool to help tackle oral health inequalities through targeted oral health promotion in areas of high need.

- The majority of dental practices in England are not proactively partaking in National Smile Month via Twitter.

- The method outlined can be applied to many other public health policy settings.

\section{Introduction}

National Smile Month (NSM) is an annual oral health campaign organised by The Oral Health Foundation (OHF), which is an independent charity working to improve oral health around the world. OHF's goal is to improve people's lives by reducing harm caused by oral 
diseases. ${ }^{1}$ Poor oral health is an important public health problem in England, costing the NHS England nearly £3.4 billion annually, despite the disease being largely preventable. ${ }^{2}$ Regional inequalities in general health across the UK are well documented and there is significant evidence to show that oral health and general health are interlinked. ${ }^{3,4}$ Inequalities in oral health are often unjust, related to social status, wealth and education. 5,6 Every year the OHF aims to help people improve their oral health by providing educational materials and resources, advice and information and by organising campaigns and events. One of the OHF's longest and most recognised campaign is NSM. ${ }^{7}$ What began in 1977 as the 'Smile 77' project, which was a week-long campaign is now the largest and longest running oral health campaign in the United Kingdom and is aimed at educating and engaging people about the importance of good oral health. Through NSM, the Foundation aims to work more closely with oral health educators, health professionals, schools and workplaces, to increase the delivery of oral health education, especially in disadvantaged communities and regions of known poor oral health. The campaign aims to encourage participates to promote good oral health through organised events and interventions in their local community. ${ }^{7} \mathrm{NSM}$ incorporates several social media initiatives alongside the event including running a Twitter feed linked to the campaign.

Twitter is a popular social media application for online news and social networking and is about discovering interesting and influential people and organisations online by following their burst of messages. Twitter encourages people and organisations to tweet (to communicate) their opinions and ideas on events and current affairs without barriers in 140 characters (NB, this character limit has since been increased to 280 characters in November 2017). Tweets can contain video, photos and links as well as text. It is proposed that Twitter has 330 million active users 'tweeting' about breaking news, entertainment, sports, politics, and everyday interests. Twitter is popular among celebrities and politicians, and the use of Twitter has become commonplace through many nations in self-promotion, advertisement and to influence as well as in political discussion. ${ }^{8}$

When it comes to health campaigns social media platforms including Twitter have been used successfully to encourage those seeking support to tackling obesity, ${ }^{9}$ Slimming World has attracted thousands of people to engage in their online app to share stories, tips and to give advice and support. Similarly, to improve the wellbeing of new mums, ${ }^{10}$ Netmums has a huge following and is now in the Top 50 websites and blogs for new parents. Twitter has also been used to decrease experience of isolation in people suffering from chronic pain, through online communities that 
share and discuss their experiences and give support. ${ }^{11}$ Collectively, these campaigns and blogs reveal that social media can be highly effective in raising awareness and motivating participants to make small changes. Through Twitter, participants of NSM are encouraged to access the latest updates on the campaign by following the Oral Health Foundation's Smile Month (@nationalsmilemonth) and then become involved on Twitter by using specific hashtags (a short keyword prefixed with a \#). Tweets on Twitter are public unless users specifically mark their tweets as private, and thus creates a rich source of information on beliefs, attitudes, reactions and opinions from a broad range of people. The campaign also uses other forms of social media such as Instagram and Facebook, to encourage people to get involved and spread positive oral health messages.

The use of social media in public dental health campaigns supports the view expressed by Sheiham \& Watt (2000) who stated that the role of the dentist in the twenty first century should no longer be merely to concentrate on the 'one to one' aspect of patient care but to take into consideration the wider community. ${ }^{12}$ Research is now shedding light on ways to reduce health inequalities through workforce innovations that increase access to preventative health care services. ${ }^{13-15}$ Many of these innovations recognise the need for promotion of dental services outside the traditional dental-office environment, in non-dental community-based settings and targeting 'hard to reach' groups. ${ }^{14}$ Thus, it has been suggested that health communicators should step further into social media engagement and embrace the 'social' nature of social media which is 'all about "virtual" engagement', participation and relationship building. ${ }^{15}$ However, how Twitter is currently being used in oral health promotion and the benefits to the population's oral health has been underexplored.

At the time of this research, there were very few studies that had explored the role of Twitter in a health campaign. A literature search of electronic bibliographic databases was undertaken in August 2018 to identify any relevant research published since the initial search in March 2016. Using the EBSCO Discovery Database a 'social sciences' search was conducted using the following 4 keywords 'Twitter' 'health promotion' 'regional inequalities' and 'oral health'. The search revealed that there have been several studies that have explored the use of Twitter as a tool for health promotion. For example, McClellan, et al (2017) researched the use of Twitter to monitor mental health discussions, ${ }^{16}$ Gough et al investigated the ability of Twitter to induce attitude and behaviour change in skin cancer prevention ${ }^{17}$ and Gabarron et al (2018) ${ }^{18}$ examined the 
use of social media for health promotion in diabetes. Chung (2017) ${ }^{19}$ analysed the sharing of tweets (retweeting) of health messages, specifically around Breast Cancer Awareness Month. However, the search did not reveal research that specifically explores Twitter's ability to address dental health through health promotion or regional health inequalities. However, all these studies reveal that Twitter and social media are quickly becoming important tools for improved public health communication

The purpose of this research was to understand how effectively Twitter was being used by dentists and dental care professionals during NSM and whether it had the potential to address regional oral health inequalities by answering the following questions -

1. What was the frequency of tweets received during NSM from dental practices, oral health educators and organisations in England?

2. What was the content of these tweets?

3. What was the reach and impact of these tweets across different socio-economic geographies?

\section{Methods}

The research involved a cross-sectional study and used a mixed method approach as the influence of a Twitter campaign can be conceptualised as both extensive, relating to a quantitative analysis of the spread and reach of the campaign, and intensive, referring to the qualitative assessment of the campaign's impact and the participant's reaction to it. ${ }^{20}$

Twitter was chosen as the most appropriate social media platform for this research as most social media activity by health professionals happens on Twitter. ${ }^{21}$ Twitter is a free and popular platform with an open and accessible application processing interface (API). An API is a set of functions and procedures that allow the creation of applications which access the features or data of an operating system, application, or other service. Because of the platform's simplicity, developers have been able to create many easy to use online applications for social scientists which enable the capture and collection of tweets from Twitter. The Twitter hashtag enables sorting and analysis of data using keyword searches. Due to the 140 character word limit at the time of the research, content analysis was relatively straightforward. Tweets can be collected in 'real time' thus producing a time series analysis of the frequency of Tweets over a set period of time. Other social media platforms currently do not have these applications that enable simple methods to gather such a rich source of information. 
To measure the frequency of Twitter posts (i.e. 'tweets') linked to the campaign, tweets were collected using a free Windows program for keyword content analyses of social media texts (Mozdeh - http://mozdeh.wlv.ac.uk/). Mozdeh was used to collect data for one month during the NSM campaign from 15/05/16 to 16/06/16 using the following key words that were related to the campaign - \#MySmileySelfie, \#NSM16, \#NSM, @smilemonth and \#nationalsmilemonth16.

To measure reaction, a content analysis of the tweets was performed and tweets were manually classified based on Neiger's ${ }^{22}$ model of social media engagement. Neiger proposed an evaluation hierarchy and applied the developed hierarchy to a hypothetical promotion campaign using Twitter as the main campaign vehicle. Neiger proposed that engagement types can be classified into three stages - Low, Medium and High.

Examples of the classified Tweets using this hierarchy can be viewed in Table 1

(Table 1)

Table 1. Classification of tweets and examples

\begin{tabular}{|l|l|l|}
$\begin{array}{l}\text { Classification } \\
\text { of tweets }\end{array}$ & Indicators & Example \\
Low & $\begin{array}{l}\text { One-way closed messages, devoid } \\
\text { of educational information, self- } \\
\text { promoting }\end{array}$ & $\begin{array}{l}\text { We are really happy to be } \\
\text { celebrating @smilemonth with } \\
\text { activities at the practice. \#smiles } \\
\text { \#nsm16 }\end{array}$ \\
$\begin{array}{l}\text { Beginning of dialogue. Engaging, } \\
\text { encouraging a response/reaction } \\
\text { educational information, } \\
\text { infographics, links to blogs and } \\
\text { healthy tips }\end{array}$ & $\begin{array}{l}\text { Advice on sugar and children's } \\
\text { oral health to keep their teeth in } \\
\text { tip top condition! Ready for }\end{array}$ \\
$\begin{array}{l}\text { @smilemonth! } \\
\text { \#nationalsmilemonth16 }\end{array}$ \\
$\begin{array}{l}\text { Activities organised as a direct } \\
\text { result of the campaign outside of the } \\
\text { virtual environment with direct } \\
\text { benefits to the local community }\end{array}$ & $\begin{array}{l}\text { \#MySmileySelfie - OHP team } \\
\text { promoting NSM at Asda } \\
\text { Blackburn \#nsm16 }\end{array}$ \\
\hline
\end{tabular}

To explore whether NSM was being used by dental practices to engage communities in areas of high dental need through events linked to promoting good oral health, the 
postcodes from the tweets classified as high were manually collated from the website link in the user's Twitter profile. The Department for Local Communities and Government Postcode Lookup tool was used to obtain deprivation data and the Lower Super Output Area (LSOA) code in which each postcode was located. ${ }^{23}$

The choropleth map is a useful tool that can emphasise visually relationships between different variables i.e. deprivation and a geographical location. In order to produce a visual display of the data, choropleth maps were developed using digital boundary data obtained from the Office for National Statistics in a shapefile which contained the information for mapping the Local Authority boundaries of England. A shapefile is a simple format for storing the geometric location and attribute information of geographic features. The shapefile was then converted into a Keyhole Markup Language file (KML) using Shape Escape (www.shpescape.com) and imported directly into Google Fusion Tables (GFT). GFT is a powerful mapping tool that enables the user to create interactive maps, it maps data from postcodes (geocode) or geospatial information via KML files. Keyhole Markup Language (KML) is an encoded notation for expressing geographic annotation and visualization within Internet-based, two-dimensional maps and three-dimensional Earth browsers.

In order to explore the association of tooth decay in children and Twitter engagement the tweets classified as 'high engagement' and the data from the Public Health England's 'Dental Public Health Epidemiology Programme' 2014/15 (DPHEP) oral health survey of 5 year olds was collated. ${ }^{24}$ The data were then merged with the shapefile data into GFT. To create the colour gradient in the choropleth map, the data were split into quintiles (buckets) that were matched to the percentage of decay in 5 year olds. The higher the percentage in that location the darker the colour.

To display the location of the tweets classified as 'high' engagement on the map, the postcodes of where the tweets originated from were uploaded into the GFT. The GFT automatically recognises locational information such as postcodes and will geocode the data and display a marker in the correct geographical area.

The same process was conducted for the map created for the Indices of Multiple Deprivation (2015) data which is captured at the Lower Super Output Area (LSOA) level. The information required for the GFT was obtained using the data that Alasdiar Rae 
(Sheffield University) ${ }^{25}$ created for public use by merging the geographical boundaries of England with the Indices of Multiple Deprivation 2015 data.

In order to visibly explore the association between neighbourhood deprivation, levels of tooth decay in 5 year olds and the location of those dental practices/organisations tweets classified as 'high engagement' in NSM the Google Fusion Layer Wizard web application was used. The application enabled the layering of the two maps created from the 'high' classified participant's postcode (47 tweets) and the interactive choropleth maps of deprivation and the data from the DPHEP 2014/15 survey of decay in 5 year olds. All the maps created were interactive so they enabled the user to reveal more details of a specific geographical location. Figure 1 shows the association between deprivation, decay levels and Twitter activity classified as 'High' in that region.

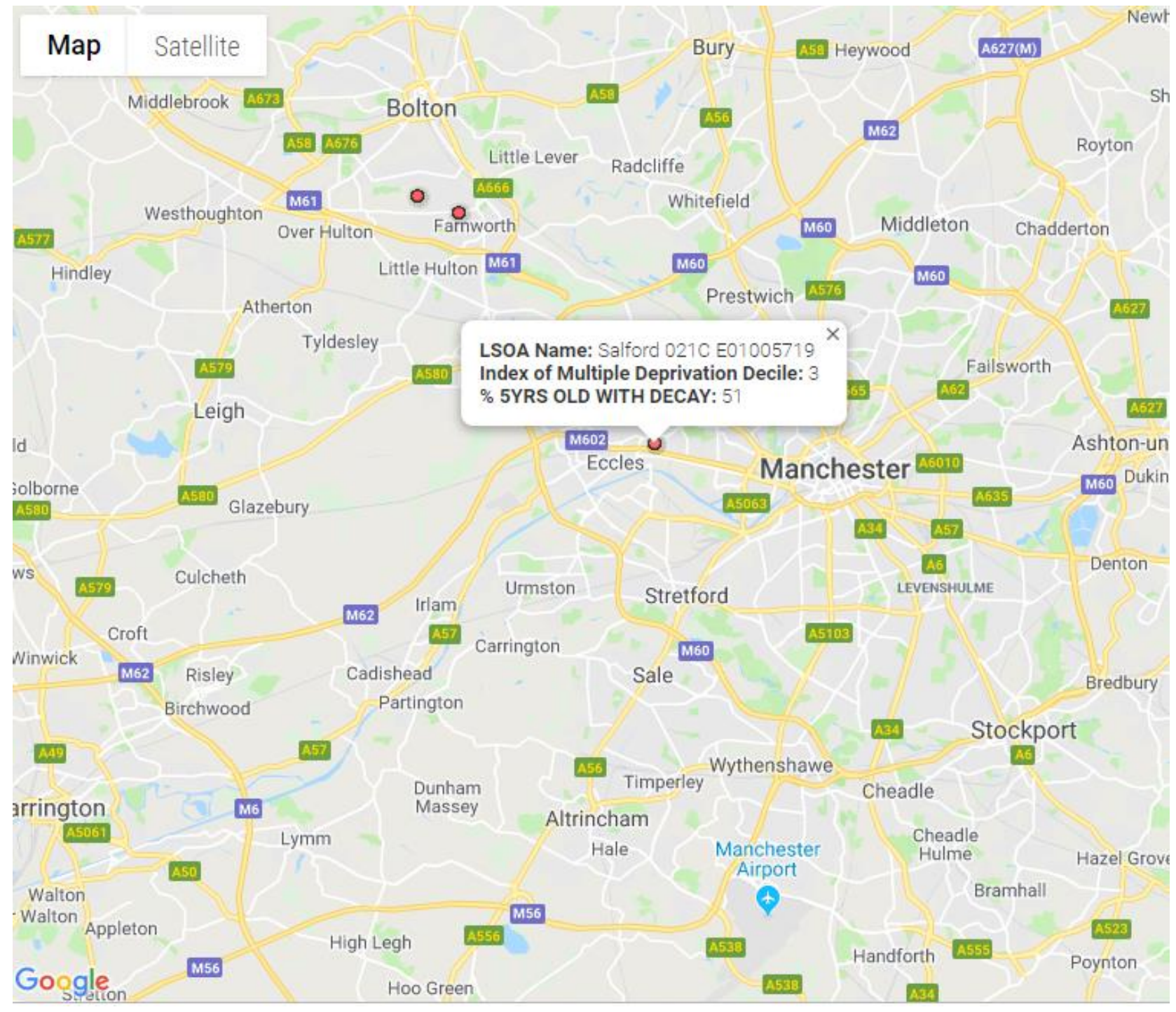

Figure 1: Association between 'high' engagement, deprivation and level of decay in 5 year olds 


\section{Results}

A total of 23100 Tweets were collected over the duration of the campaign. Once the marketing tweets, tweets from individuals and unrelated tweets were removed, there were a total of 2968 usable tweets from 763 separate Twitter accounts. 212 were from dental practices. 107 of these were classified as low engagement, 99 medium, and 6 high according to the system outlined in table 1. As the level of tweets classified as 'high' from dental practices was so low it was decided to include the high classified tweets from other publicly funded organisations allied to oral health during NSM 2016; such as NHS Oral Health Promotion teams and Local Authority Wellbeing teams. This gave an extra 39 tweets equalling a total of 45 tweets classified as high.

Figure 2 below shows the volume of tweets received from all participants over the NSM month which included individuals, organisations and businesses.

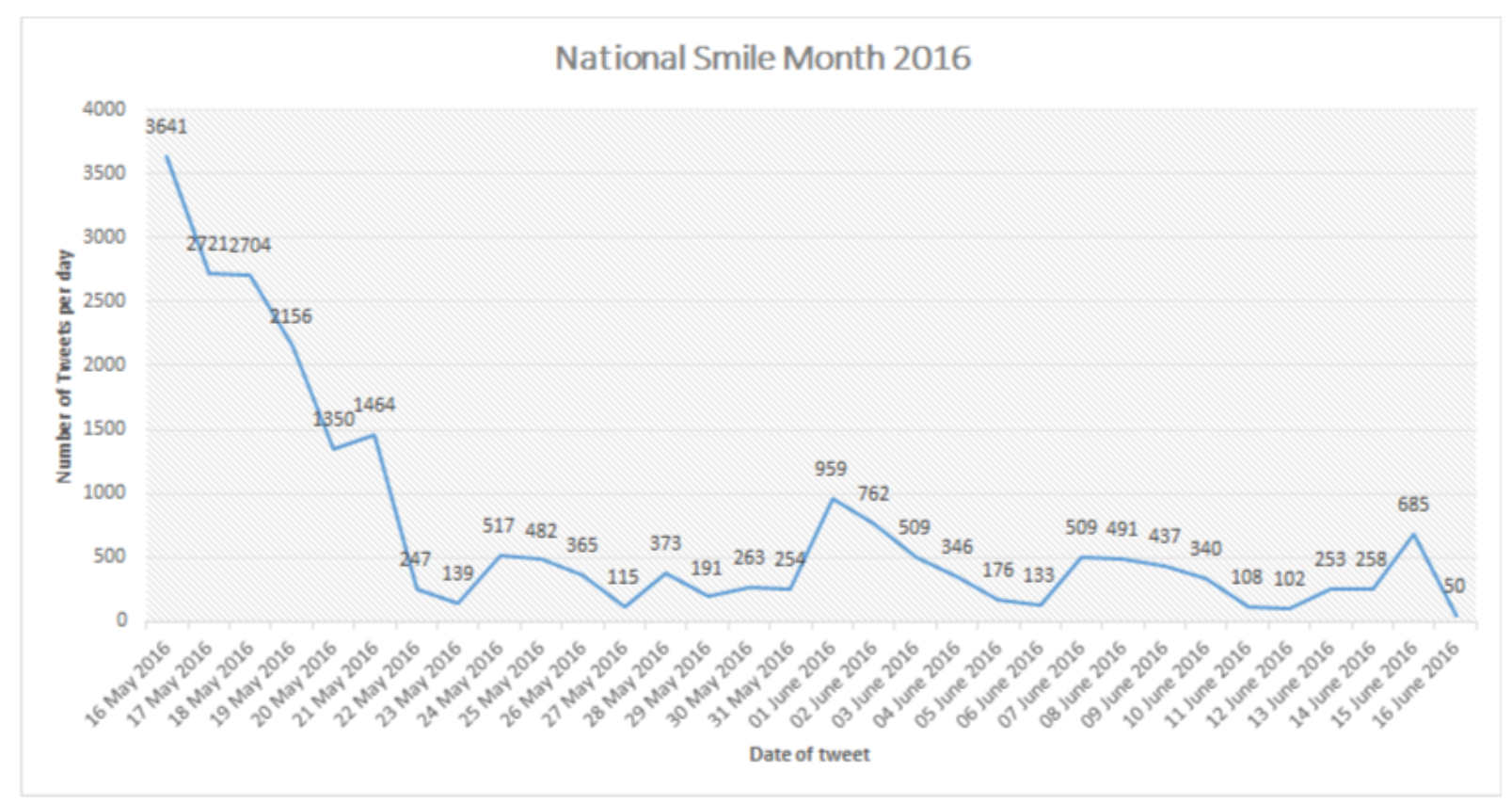

Figure 2. Frequency graph of tweets (May $16^{\text {th }}$ to June $16^{\text {th }}$ 2016)

The Twitter activity followed a classic decay curve, showing that initial interest in tweeting about NSM was popular at the start of the campaign but then the number of daily tweets tapered off quickly (Figure 2). 3641 Tweets were captured on the first day of the NSM Campaign, that declined steadily and by the second week on the $23^{\text {rd }}$ of May only 139 Tweets were captured. There was a slight increase in Tweets from the $31^{\text {st }}$ May that 
peaked on the $1^{\text {st }}$ of June during the 2016 school half term when 959 Tweets were captured.

To explore the relationship between the Tweets classified as 'High', neighbourhood deprivation and decay in 5 year olds a Google Map was created using GFT. Figure 3 is a choropleth map that was created as the base map; this is a thematic map which is shaded in relation to the percentage of decay in 5 year olds, the darker the blue the higher the decay rates.

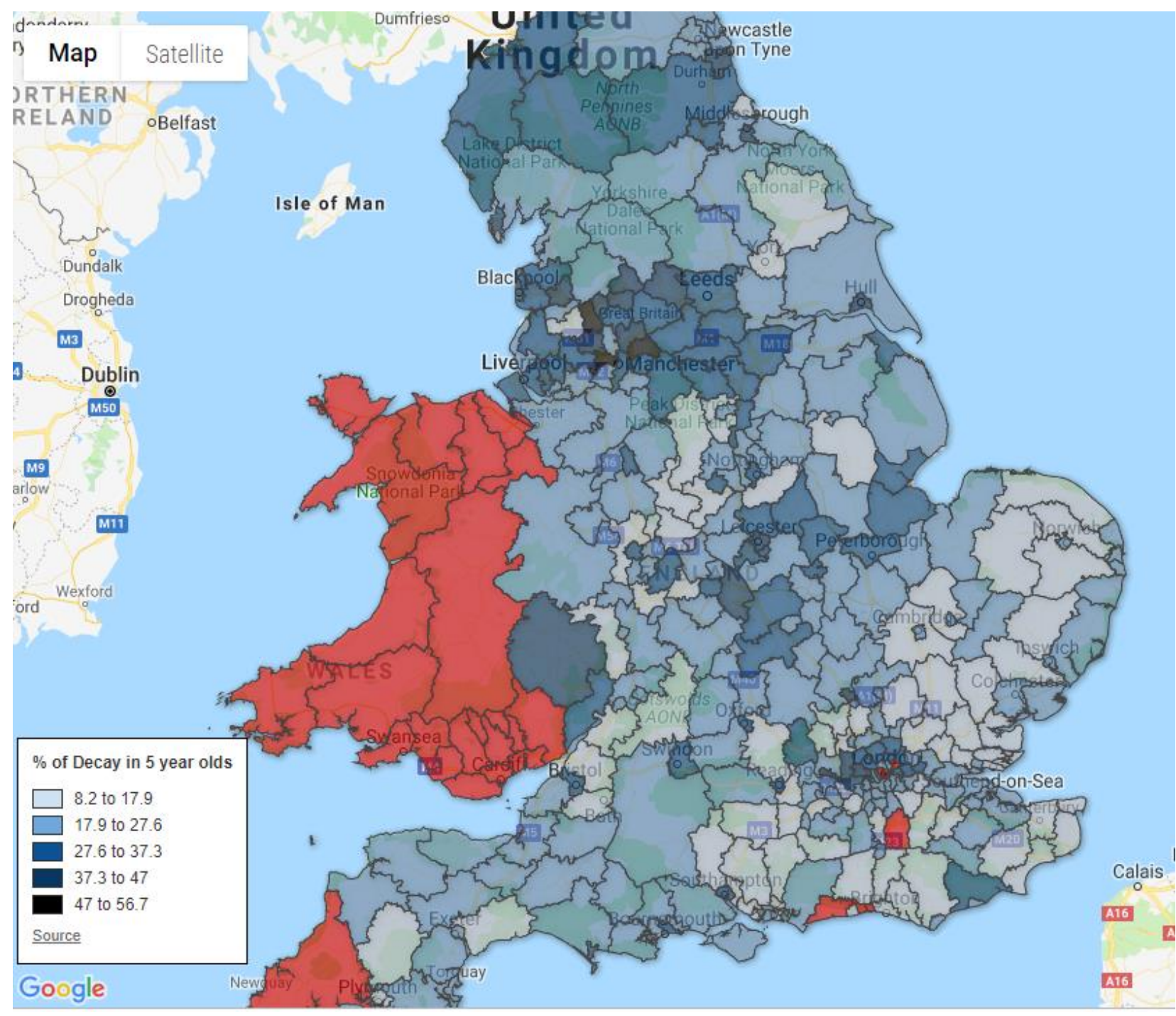

Figure 3; Percentage of decay in 5 year olds (red areas = no data) 


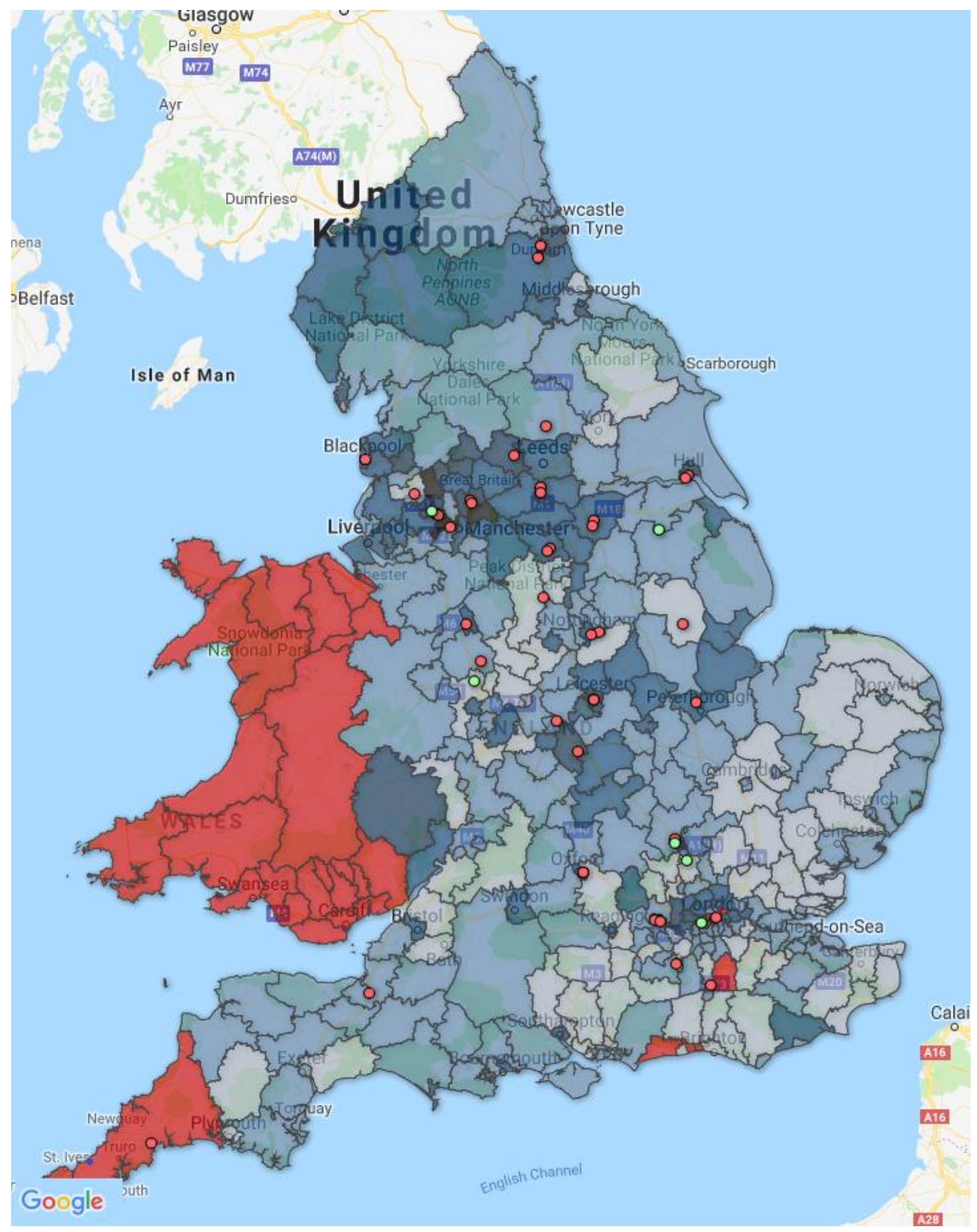

Figure 4; Layered map depicting the relationship between decay in 5 year olds, Indices of multiple deprivation and geolocation of high tweets 
This map (Figure 4) was generated by layering four google fusion tables 1) base map DPHEP survey of decay in 5 year olds (Figure 3) 2) Indices of multiple deprivation index 3) High classified tweets from dental practices (green markers) 4) High classified from organisations allied to oral health (red markers).

The map (Figure 4) provides a visual representation of the area's deprivation rank and level of decay in 5 year olds. The locational marker (red and blue dots) of tweets classified as 'high' during NSM 2016 depict the relationships between the geographic location level of deprivation and the percentage of decay in 5 year olds. The map illustrates that local authorities across England are engaging more with partners to tackle oral inequalities More NSM community events via Twitter were organised in deprived areas of high decay in 5 year olds.

\section{Discussion}

This research analysed the engagement via Twitter in NSM from dental practices and organisations to help address oral health inequalities. Twitter is currently the most popular microblogging social media platform and is a useful way of bringing together like-minded communities that share a common goal. ${ }^{26}$ Despite this, the tweets captured during the NSM campaign 2016 indicated that participation on Twitter was mainly superficial (classified low and medium engagement) and used as a one-way communication/recruitment tool by dental practices in NSM, which alone will not help towards tackling oral health inequalities. There is a psychological explanation for this type of behaviour. Online viral campaigns have been said to mainly leverage 'extrinsic' incentives to do 'good' such as following NSM, tweeting or re-tweeting information, rather than cultivating an internally sourced 'intrinsic' motivation to help others in the real world. ${ }^{27}$ This behaviour was evidenced in the initial peak in tweets followed by a steady decline. This trend is comparable to results reported in research exploring the use of Twitter to raise awareness of Breast Cancer. ${ }^{28}$ In other words, the optimistic use of social media to propel positive social change is balanced by the shallow, short lived nature of engagement with anything 'viral'. The majority of "viral" campaigns draw their success from the psychology of 'consensus', (i.e the number of followers tweets and re-tweets) but the nature of NSM requires a deeper engagement such as activities outside of the virtual environment. Research has shown that activities which engage and educate people on oral health 'outside' of the virtual environment are more likely to have impact in the local community. ${ }^{29}$ Any real effort to help address oral health inequalities would require dental 
practices to engage in activities that promote good oral health outside of their clinical walls, such as street campaigns, local fetes and events and outreach services for the hard to reach population. Many Local Authorities, who are now responsible for public health in their areas are attempting to better meet the needs of their local population through evidence-based approaches such as water fluoridation schemes, community based fluoride varnish schemes and supervised tooth brushing in early years' settings. ${ }^{30}$

The number of dental practices registered in England was 10,130 in March 2015 and there are estimated to be 32,900 dentists working in general dental practice in the UK and 43,350 registered dental care professionals. ${ }^{31}$ Only 201 dental practices participated in NSM 2016 via Twitter, that is only 2 percent (1.98\%) of dental practices in England engaging in NSM via Twitter.

A British Dental Association (BDA) report in 2008 suggested that the 2006 NHS contract has created unfair pressures and uncertainty for dentists with around 500 dentist lost within the first year of the new system. ${ }^{32}$ This consequential uneasiness and anxiety has done little to encourage a greater number of dentists to work for or devote more time to NHS dentistry. In fact recent responses to a BDA survey in 2017 suggest that $58 \%$ of young dentists intend on leaving the NHS. ${ }^{34}$

To address oral health inequalities targeted dental practices in areas of high need/deprivation could be given the resources and financial incentives to be more proactive in campaigns like NSM. A more balanced distribution of efforts and resources along with the full range of intervention points from the downstream curative to the upstream structural healthy policy is the goal to successfully working towards tackling oral health inequalities. ${ }^{5}$ The NSM campaign encourages dental health professionals to establish links with homeless shelters, community and day care centres, residential homes and hostels during NSM month, these relationships are valuable in helping to address regional inequalities. Local Authorities and their healthcare partners could consider collaborating more during NSM with dental practices to help spread the 'prevention' message. 


\section{Limitations}

The results of the research should be interpreted according to the following limitations. This research only takes into the account the Twitter element of NSM. Many dental practices may not use Twitter and/or could have participated in the campaign with activities, talks and displays outside the realms of social media.

Mozdeh an API Search software was used to collect the tweets. Twitter states that the Search API is "focused on relevance and not completeness. This means that some Tweets and users may be missing from search results. If you want to match for completeness you should consider using a Streaming API instead" - [https://developer.twitter.com]. Subsequently, this data should only be considered a 'snapshot' of the Twitter activity surrounding NSM. It is also possible that the number of tweets during NSM was underestimated because they were not covered by the selected key words. The postcodes captured for this data were linked to the tweet and not the actual event itself. Some of the Tweets were lost due to 'unmatched' data in the postcode lookup tool.

The data from the Dental Health England Epidemiology study of 5 year olds ${ }^{24}$ survey was conducted at Local Authority (LA) lower tier level and not at the Lower Super Output Area level. Many LA districts can have areas of affluence and areas of deprivation; this will affect the overall estimates of disease trends. The Dental Health England Epidemiology survey of 5 year olds (DPHEP) also relies on an 'opt in' basis and this relies on parents/guardians completing a consent form for their child to take part in the survey. This may lead to potential under representation of lower socio-economic groups as some sections of the community are more likely to provide positive consent than others. The survey examined the dental health of 111,500 children whose parents and carers 'opted in' to the survey.

Notwithstanding the limitations, this research, for the first time has developed a simple method in which to evaluate the geographic reach of an online campaign. All the tools needed to replicate similar research of an online campaign can be done using free open source web applications. Very little research has been conducted in the value of social media as a tool to tackle oral health inequalities. The use of social media to help tackle oral health inequalities is largely unexplored and contributes to the originality of this research. 


\section{Conclusions}

Engagement with NSM 2016 on Twitter was mostly superficial and did not raise awareness the importance of improved oral health via this social media platform. The results from this research indicated that dental practice teams are not fully engaging with social media in a way that would encourage good oral health to the population beyond their surgery. Of the dental practice teams which did engage with NSM, the majority of their tweets did not promote any specific preventative behaviour. The research provides evidence that publicly funded organisations allied to oral health were more pro-active in NSM via Twitter by targeting their NSM activities in areas of high need. To increase more meaningful participation in NSM from dental practices the NHS needs to create a dental service that financially rewards dentists for the implementation of oral health promotion that is inclusive for the whole community. Local Authorities could work more closely with their healthcare partners and local dental practices to improve the oral health of the local population through interventions and community based programmes and events.

These maps are intended to be viewed online and a folder containing the Google Fusion Tables and Maps can be found by cutting and pasting this link into your browser: https://drive.google.com/open?id=1 V4t ZRet1bY 3gX5ze5ljHZ4eeEUyOmH

Use Google Layer Wizard to overlay the maps https://developers.google.com/maps/documentation/javascript/layers\#FusionTables 


\section{References}

1. Oral Health Foundation | Home. https://www.dentalhealth.org/. Accessed August 21, 2018.

2. Improving Dental Care and Oral Health - A Call to Action. 2014;(February):1-34.

3. Locker D. Concepts of oral health, disease and the quality of life. Measures of Oral Health-related Quality of Life. 1997:172.

4. Petersen PE. The World Oral Health Report 2003: continuous improvement of oral health in the 21 st century--the approach of the WHO Global Oral Health Programme. Community Dent Oral Epidemiol. 2003;31:3-24. doi:10.1046/j.2003.com122.x

5. Watt RG. From victim blaming to upstream action: Tackling the social determinants of oral health inequalities. Community Dent Oral Epidemiol. 2007;35(1):1-11. doi:10.1111/j.1600-0528.2007.00348.x

6. Review TM. Fair Society Healthy Lives. Nursing Standard. 2010;25(6):30-30 . doi:10.7748/ns2010.10.25.6.30.p4603

7. Oral Health Foundation | National Smile Month. https://www.dentalhealth.org/whatis-national-smile-month. Accessed August 21, 2018.

8. Bastos MT, Zago G. Tweeting news articles. Readership and news sections in Europe and the Amercias. SAGE Open. 2013;3(3):215824401350249. DOI:10.1177/2158244013502496

9. Dickins M, Thomas SL, King B, Lewis S, Holland K. The role of the fatosphere in fat adults' responses to obesity stigma: A model of empowerment without a focus on weight loss. Qual Health Res. 2011;21(12):1679-1691.

doi:10.1177/1049732311417728

10. McDaniel BT, Coyne SM, Holmes EK. New mothers and media use: Associations between blogging, social networking, and maternal well-being. Matern Child Health 
11. Ressler PK, Bradshaw YS, Gualtieri L, Chui KKH. Communicating the experience of chronic pain and illness through blogging. J Med Internet Res. 2012;14(5):e143. doi:10.2196/jmir.2002

12. Sheiham A, Watt RG. The common risk factor approach: a rational basis for promoting oral health. Community Dent Oral Epidemiol. 2000;28(6):399-406. doi:10.1034/j.1600-0528.2000.028006399.x

13. Petersen PE, Kwan S. Equity, social determinants and public health programmes the case of oral health. Community Dent Oral Epidemiol. 2011;39(6):481-487. doi:10.1111/j.1600-0528.2011.00623.x

14. Caton S, Greenhalgh F, Goodacre L. Evaluation of a community dental service for homeless and "hard to reach" people. Br Dent J. 2016;220(2):67-70. doi:10.1038/sj.bdj.2016.56

15. Newbold B. 2014 Engaging communities through social media: Effectiveness, impact, and outcomes engaging communities through social media: University at Buffalo, New York. October 29th 2014. http://ktdrr.org/conference2014/expo/materials/102914.Newbold.pdf

16. Mcclellan C, Ali MM, Mutter R, Kroutil L, Landwehr J. Using social media to monitor mental health discussions evidence from Twitter. doi:10.1093/jamia/ocw133

17. Gough A, Hunter RF, Ajao O, et al. Tweet for behavior change: Using social media for the dissemination of Public Health messages. JMIR Public Heal Surveill. 2017;3(1):e14. doi:10.2196/publichealth.6313

18. Gabarron E, Bradway M, Fernandez-Luque L, et al. Social media for health promotion in diabetes: study protocol for a participatory public health intervention design. 2018. doi:10.1186/s12913-018-3178-7

19. Chung JE. Retweeting in health promotion: Analysis of tweets about Breast Cancer 
Awareness Month. Computers in Human Behaviour. 2017;74:112-119.

doi:10.1016/J.CHB.2017.04.025

20. McNeill A. Theoretical perspectives on the use of social media in the propagation of health messages. :3-6.

https://hcihealthcarefieldwork.files.wordpress.com/2013/11/theoryhealthchi2014_sub mission_2.pdf

21. Kass-Hout TA, Alhinnawi H. Social media in public health. Br Med Bull. 2013;108(1):5-24. doi:10.1093/bmb/ldt028

22. Neiger BL, Thackeray R, Burton SH, Giraud-Carrier CG, Fagen MC. Evaluating social media's capacity to develop engaged audiences in health promotion settings: Use of Twitter metrics as a case study. Health Promotion Practice. 2012;14(2):157162. doi:10.1177/1524839912469378

23. English Indices of Deprivation 2015: Postcode Lookup. http://imd-bypostcode.opendatacommunities.org/. Accessed August 21, 2018.

24. Dental Health Survey Results. http://www.nwph.net/dentalhealth/survey-results 5(14_15).aspx. Accessed August 28, 2018.

25. Deprivation mapped: how you show the poorest (and richest) places in England | News | The Guardian. https://www.theguardian.com/news/datablog/2011/mar/31/deprivation-map-indicesmultiple. Accessed August 21, 2018.

26. Kwak H, Lee C, Park H, Moon S. What Is Twitter, a Social Network or a News Media? http://bit.ly. Accessed August 17, 2018.

27. Van der Linden S. The nature of viral altruism and how to make it stick. Nat Hum Behav. 2017;1:0041. doi:10.1038/s41562-016-0041

28. Thackeray R, Burton SH, Giraud-Carrier C, Rollins S, Draper CR. Using Twitter for breast cancer prevention: an analysis of breast cancer awareness month. BMC 
29. Petersen PE, Kwan S. Evaluation of community-based oral health promotion and oral disease prevention--WHO recommendations for improved evidence in public health practice. Community Dent Health. 2004;21(4 Suppl):319-329.

30. Health Matters: Tackling child dental health issues at a local level - Public health matters. https://publichealthmatters.blog.gov.uk/2017/06/19/health-matters-tacklingchild-dental-health-issues-at-a-local-level/. Accessed August 31, 2018.

31. Number of Dentists in the UK - Office for National Statistics.

https://www.ons.gov.uk/aboutus/transparencyandgovernance/freedomofinformationf oi/numberofdentistsintheuk. Accessed August 21, 2018.

32. BRITISH DENTAL ASSOCIATION Evidence to the Doctors' and Dentists' Review Body. 2007. https://bda.org/about-the-bda/campaigns/Documents/ddrb/200809_bda_evidence.pdf (July 25, 2019).

33. Twitter. Developers:Tap into what's happening. Available at https://developer.twitter.com/ (accessed July 2019)

34. Half of NHS young dentists heading for the exit (October 2017). Available at: https://bda.org/news-centre/press-releases/half-of-nhs-young-dentists-heading-for-the-exit (Accessed: July 2019). 